\title{
Network PerformanCE ENHANCEMENT WiTH OPTIMIZATION SENSOR Placement IN WiRELESS SENSOR NETWORK
}

\author{
Gholamreza Farahani \\ Department of Electrical Engineering and Information Technology, Iranian Research \\ Organization for Science and Technology (IROST), Tehran, Iran
}

\begin{abstract}
From one side, sensor manufacturing technology and from other side wireless communication technology improvement has an effect on the growth and deployment of Wireless Network Sensor (WSN). The appropriate performance of WSN has abundant necessity which has dependent on the different parameters such as optimize sensor placement and structure of network sensor. The optimized placement in WSN not only would optimize number of sensors, but also help to reach to the more precise information. Therefore different solutions are proposed to reduce cost and increase life time of sensor networks that most of them are concentrated in the field of routing and information transmission. In this paper, places which they need new sensors placement or sensor movements are determined and then with applying these changes, performance of WSN will calculate. To achieve the optimum placement, the network should evaluate precisely and effective criteria on the performance should extract. Therefore the criteria should be ranked and after weighting with using AHP algorithms, with use of Geographical Information System (GIS), these weighted criteria will combined and in the locations which WSN doesn't have enough performance, new sensor placement will create. New proposed method, improve $21.11 \%$ performance of WSN with sensor placement in the low performance locations. Also the number of added sensor is $26.09 \%$ which is lowest number of added sensors in comparison with other methods.
\end{abstract}

\section{KEYWORDS}

Sensor, Wireless Sensor Network, Geographic Information System, Network performance, Analytical Hierarchy process, Overlap Index

\section{INTRODUCTION}

Growth and progress of sensor technology and wireless communication causes movement to the new generation of networks named WSN. Sensor placement in WSN is one of the important issues in network design and implementation. Therefore determination of sensor placement strategy is an essential step in design and optimization of network. As it is clear, optimum placement of sensors lead to increment and enhancement of WSN performance. There are many map points which it could be used to install sensors there that it will made a complicated problem, also all of these map points will not improve the network performance. To increase network performance, it requires considering different parameters to find the optimum place. For sensor placement, the network should study carefully and extract effective parameters which with help of these parameters, some sensors will install in locations that require new sensors. Geographical Information System (GIS) is a set of combination of computer hardware and software, spatial 
information and experts which designed and created for storage, update, processing, analyze and presentation of spatial information.

Reference [1] achieves a sensor placement in water distribution network with use of ant colony algorithm. In this paper with consideration limited number of sensors and limitation such as cost, physical condition of sensors and possibility or impossibility of sensor installation between nodes, proper location of sensors is chosen. Reference [2] proposed a placement of Tehran city pollution measurement stations that with use of population, they determined stations and location of them, also it proposed new stations with using stations measurement to obtain a better performance. Reference [3] presents an algorithm for placement of disaster management sites. In this algorithm with use of effective criteria and AHP method, locations for new disaster management sites are proposed. Reference [4] proposed a program to solve a WSN problem to obtain a network optimal configuration. The aim of this paper is finding a set of optimum placements to install sensors and base stations to recover connection and covering requirements. The parameters which are considered for this work were measurement range and field of view. Reference [5] proposed a method for sensor placement and optimization of related variables in water distribution system based on different scenarios and different optimum variables. They analyzed distribution of network sensor location and with use of spatial information system; the best sensors placement is calculated. Reference [6] presents a new strategy to determine optimal sensor placement in WSN. This will carry out by minimization of spatial analysis of variance method (ANOVA) base on selection of random points to determine sensor locations. Spatial analysis is carried out with use of geostatistic analysis and optimization with Monte Carlo analysis. Reference [7] presents a method for sensor implementation and programing to increase sensors coverage. They used a bee algorithm to find a sensor placement and shown use of bee algorithm will provide worthy results. Reference [8] states that different optimum algorithms are developed and used which most of these algorithms are based on sensor models and simple network. They don't use environment spatial information such as earth model. Therefor to implement an effective sensors network, it proposed sensor network deployment with considering sensor spatial information and obtained optimum sensor placement.

In optimization of sensors placement, important parameters which are effective on the sensor placement such as sensors lifetime, sensor coverage area, slope, slope direction, distance from adjacent node and distance from base station should be extracted. After parameter extraction, these parameters should be delivered to experts on the sensor network to determine importance degree of them. After it, with use of AHP algorithm, weight of each parameter related to the WSN performance should be calculated. Then with GIS, the locations of network which have low performance will determine and new sensors will place on those locations to obtain a better WSN performance.

\section{EFFECTIVE PARAMETERS IN SENSORS NETWORK}

Several factors are involved in placement of sensors on the WSN that the most important which has very effect in the sensors location are bring at continuation.

\subsection{Network Structure}

A sensor network can be built as hierarchical or flat. In hierarchical structure some sensors are as a central sensor and collect environmental report of their neighboring sensors. The collected data will send to base station via these central sensors. In flat structure, unlike the hierarchical 
structure, role and operation of all sensors are same and information processing and sending information to base station is sensors responsibility [9]. In this paper the network structure is considered as flat.

\subsection{Coverage}

Coverage commonly defines as that how well the sensor network covers the intended region. Appropriate coverage of sensors; will cause optimum energy consumption of sensors. Generally targets in sensors network divide into three common categories, full supervisory on the region, supervisory on set of targets and intrusion detection between natural barriers and environment. Therefore coverage in sensor networks also divides to three categories regional coverage, target coverage and barrier coverage. Regional coverage means each point inside intended region is at least on the measuring range of one sensor and ideal is to cover whole region with minimum sensor. Target coverage includes observation and supervisory on the defined number of targets. Generally these type of coverage used in military applications [10-12]. Coverage evaluation according to sensor coverage model which use for commutative coverage of placed sensors is different. Therefore sensor measuring model has an important role in the evaluation and calculation of coverage. Different models are proposed for coverage. In some studies coverage of each sensor will decrease with increasing the distance from sensor. Equation (1) shows sensor sensitivity values $S$ of sensor $S_{i}$ at point $P ; d\left(S_{i}, P\right)$ is the Euclidean distance between sensor and the point, $\lambda$ is a positive parameter and $\gamma$ is between 2 to 5 related to environment features [13].

$$
S\left(S_{i}, P\right)=\frac{\lambda}{\left[d\left(S_{i}, P\right)\right]^{\gamma}}
$$

Other method is binary code which is used in this paper. In this model for each sensor a coverage radius is considered and environment will convert into binary clusters. Points inside of the range are covered points and points outside the range are outsight points. This model also named disc model. Equation (2) shows a binary model which $C\left(S_{i}\right)$ is $S_{i}$ sensor coverage with distance d from point $P$ and $r_{S}$ is coverage distance [14].

$$
C\left(S_{i}\right)=\left\{\begin{array}{cc}
1 & d\left(S_{i}, P\right)<r_{s} \\
0 & \text { otherwise }
\end{array}\right.
$$

In this paper with consideration measuring range of each sensor, some buffers (circular rings around each sensor) will make. Regions which are not inside of buffers they will not be in the sensor coverage and they are coverage gaps.

\subsection{Type of Placement}

Sensor placement has an important effect on the network efficiency and sensors life time. There is different strategy for sensor placement which can be categorized into two general categories: static placement and dynamic placement. In this paper the initial sensor placement is static, although placement for new added sensors is dynamic. In static placement, first sensor placements determine randomly or planned, and then with goal of network performance improvement, new dynamic sensor placement should carry out. 
International Journal of Wireless \& Mobile Networks (IJWMN) Vol. 9, No. 1, February 2017

\subsection{Initial Sensor Placement}

Sensors in WSN can be place in two types: planned placement and random placement. In programmable implementation, sensor placement of flat architecture will choose selectively to reach the goals of specific application. Generally the goals of this design is maximum coverage range, minimum energy consumption and powerful network communication. Programmable implementation is appropriate for a wide range of WSN applications. In some environments, it is not possible to present there and install the sensor in specified location; therefore random placement of sensors in environment is only possible solution $[15,16]$.

\subsection{Energy Consumption}

WSNs are constituted with hundred or thousand nodes which are spread out in the far off or dangerous areas. Each sensor node includes sensor, an arithmetic logic unit, memory and wireless communication unit with limited range which all of these units for continuation of their works need an energy source. One of the important factors in sensor failure is finishing power supply.

Generally sensor energy is supplied with batteries which are not large and if they finish, should be changed or charged. It is important to have sensor with low energy consumption; because the sensor node should stay alive until end on its mission [17, 18]. In this paper, criterion will consider for energy consumption and if the sensor has a more energy, measuring around it will be more stable and probability of coverage gap around it will reduce.

\subsection{Network Scalability}

In sensor network, there are hundred or thousand nodes that each sensor in the necessary time should connect to the destination. In such a network, may be some nodes shut down or new nodes add to the network and network topology change. A network which tolerate these variations and continue to work, named scalable network. Therefore node density should change from a few to a few hundreds. Node density will calculate according to equation (3):

$n(R)=\frac{N \cdot \pi \cdot R^{2}}{A}$

where $n(R)$ is average number of nodes which are in the node range. In the equation (3), $A$ is working area, $N$ is number of nodes and $R$ is the radio transmission range [19].

\subsection{Digital Elevation Model (DEM)}

DEM is a digital file included elevations terrain without any extra definition about surface. DEM files could be use to determine landform, slope and other aspects of terrain. Results show elevation and slope have a destructive effect on the sensors network and whatever the slope is more, the sensor network performance will decrease. There are many equations to review effect of terrain on the sending signal over the sensor network which one the important of them is Durkin algorithm. In this algorithm, deterioration of the path included two parts. Part one of algorithm convert topographical DEM file to topographical database and reconstruct ground profile information during the path between sender and receiver. Part two of algorithm calculates expected failure during the path [20]. 


\subsection{Communication range}

Sensors have a range and radius measurement, moreover they have communication range which is not constant in some sensors and can be changed based on conditions. Generally communication range is more than sensor measurement range. As shown in figure 1, two $S_{i}$ and $S_{j}$ sensors have ability to communicate together when Euclidean distance of them be less than or equal of minimum amount of communication rang [21].

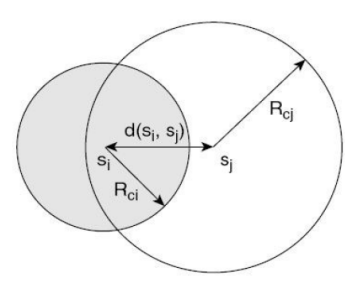

Figure 1. Communication range between two sensors

\section{MULTI-CRITERIA DECISION MAKING METHODS}

Placement is a process which many factors and criteria have impact on it. It is clear that evaluation of total factors which might help to choose best place is too hard. Therefore the model should be used that consider most effective factors on the sensor placement. Placement is decision making process with long term time, because many criteria should be tested and results of these tests use for decision and the decision to be evaluated. This evaluation could be carried out in different ways which intended criterion and aspects to be assessed simultaneously. These evaluation methods named multi-criteria decision making methods. One of the common problems in multi-criteria decision is different importance of criteria and sub-criteria for deciders. Therefore relative importance for each of these criteria and sub-criteria to one another is needed. Criteria extraction and its weighting are very important in multi-criteria decision. Each weight will insert as a number in evaluation which determine the relative importance of that criterion rather than other criteria.

There are different weighting methods to evaluate importance of criteria. Between these methods, hierarchical analysis methods are on the effective decision technique which is stated with Thomas L. Saaty in 1980 and until now it had variety applications in different sciences. This technique is based on paired comparisons and gives an opportunity to managers to compare different scenarios. Hierarchical analysis process begins with identification and prioritizing decision elements. These elements includes: goals, criteria or features and probable options which is used in priority. Elements identification process and relation between them lead to creation of hierarchical structure which is called making hierarchy. Steps of this process are as follow.

\subsection{Hierarchical Making}

In the first step, hierarchical structure of subject will determine in the diagram format. In this diagram, there is four level hierarchical includes: goals, criteria, sub-criteria and options. In hierarchical structure, analysis process of complicated problems will carry out with synthesis of it to simple details elements which are related together as hierarchical and main goal relation is identified with the lowest level of hierarchical. 


\subsection{Determination of Importance Coefficient of Criteria and Sub-Criteria}

To determine importance coefficient (weight) of criteria and sub-criteria, they compare pair wise together. Base of judgment for comparison is according to Table 1, that dominant intensity of criterion $i$ to criterion $j$ named $a_{i j}$. Pair wise comparison will record in $n \times n$ matrix. This matrix will name binary comparison criteria matrix $A=\left[a_{i j}\right]_{n \times n}$. All elements of this matrix are positive and with respect to inverse conditions principle in hierarchical analysis process, in each binary matrix, there is two numerical values $a_{i j}$ and $1 / a_{i j}$ (if significance of $i$ than $j$ is equal $k$, importance of $j$ than $i$ is $1 / k$ ). Diameter elements of this matrix, with respect to equal importance of each criterion to itself to reach the goal are one.

Table 1. Nine quantity criteria comparison

\begin{tabular}{|l|l|l|}
\hline Score & Definition & Description \\
\hline 1 & Equal importance & Two activities contribute equally to the objective \\
\hline 2 & Weak or slight & $\begin{array}{l}\text { Experience and judgement slightly favour one activity } \\
\text { over another }\end{array}$ \\
\hline 3 & Moderate importance & $\begin{array}{l}\text { Experience and judgement strongly favour one activity } \\
\text { over another }\end{array}$ \\
\hline 4 & Moderate plus & $\begin{array}{l}\text { An activity is favoured very strongly over another; its } \\
\text { dominance demonstrated in practice }\end{array}$ \\
\hline 5 & Strong importance & $\begin{array}{l}\text { The evidence favouring one activity over another is of } \\
\text { the highest possible order of affirmation }\end{array}$ \\
\hline 6 & Strong plus & \multicolumn{2}{|l}{} \\
\hline 7 & Very strong or & Extrated importance importance
\end{tabular}

\subsection{Determination of Options Importance Coefficient}

In this step, priority of each option related to sub-criteria will judge. If there is no sub-criterion for criterion, directly judge with criterion. The base of this judgment is nine quantity criteria of Saaty, with this difference that in comparison with other options related to each sub-criteria or criteria, priority intensity of option is matter not importance of it.

\subsection{Determination of Final Score Options}

In this step, by combination of importance coefficients, final score of each option will determine. For this, with using Saaty hierarchical combination principle, a priority vector by considering all judgments in all hierarchical levels will calculate as equation (4).

(priority) Final Score of $j=\sum_{k=1}^{n} \sum_{i=1}^{m} W_{k} W_{i}\left(g_{i j}\right)$

where $W_{k}$ is importance coefficient of criterion $k, W_{i}$ is importance coefficient of sub-criterion $i$ and $g_{i j}$ is score of option $j$ related to sub-criterion $i$. 


\subsection{Reviewing Compatibility in Judgments}

One of the advantages of hierarchical analysis process is capability of reviewing judgment compatibility for calculation of criteria and sub-criteria importance coefficient. When criteria significant rather others meet, there is probability of inconsistently in judgments, it means if $A_{i}$ is important than $A_{j}$ and $A_{j}$ is important than $A_{k}$, logically $A_{i}$ must be important than $A_{k}$, but unlike of all efforts, often priorities and emotions of people is inconsistent.

Mechanism which Saaty is considered for reviewing incompatibility in judgments is calculation of compatibility indices named I.R., which will calculate with dividing Incompatibility Indices (I.I.) by Random Indices (R.I.). If this indices is less or equal 0.1, compatibility in judgments are acceptable, otherwise judgments should revise. In other words the binary comparison matrix should calculate again. Incompatibility Indices will calculate as equation (5).

$$
\text { I.I. }=\frac{\lambda_{\max }-n}{n-1}
$$

where $\lambda_{\max }$ is maximum of Eigen value and $\mathrm{n}$ is number of criteria. Random Incompatibility with respect to number of criteria $(n)$ is extractable from table 2 .

Table 2. Random Incompatibility with respect to number of criteria

\begin{tabular}{|l|l|l|l|l|l|l|l|l|l|l|l|l|l|l|}
\hline $\mathbf{n}$ & 2 & 3 & 4 & 5 & 6 & 7 & 8 & 9 & 10 & 11 & 12 & 13 & 14 & 15 \\
\hline R.I. & 0 & 0.58 & 0.9 & 1.12 & 1.24 & 1.32 & 1.41 & 1.45 & 1.49 & 1.51 & 1.48 & 1.56 & 1.57 & 1.59 \\
\hline
\end{tabular}

In geometric mean method which is approximate method, instead of calculation maximum Eigen value $\left(\lambda_{\max }\right), L$ will use as equation (6).

$L=\frac{1}{n}\left[\sum_{i=1}^{n}\left(A W_{i} / W_{i}\right)\right]$

where $A W_{i}$ is a vector which obtain from multiply binary comparison criteria matrix (matrix $A$ ) by $W_{i}$ (weight vector or criteria importance indices) vector. This technique has some limitations which main of this is related to reversing scores in heirechical analysis process method . AHP assumes goals, criteria and options are in the hierechical structure and relations between them is linear and one way. This assumption always is not correct. In this condition, results of AHP method may be causes ranks reverse. Saaty presens other mehtod named Analytic Network Process (ANP) which could be used in this condition. AHP method is usefull which creates condition for analysis and conversion of complicated problems to logical and simpler hierechy that programmer could evaluate options with help of criteria and sub-criteria easily [22].

\section{GEOGRAPHIC INFORMATION SYSTEM (GIS)}

GIS is a computerized system to record, storage, edit, update, analysis and monitoring of spatial data with coordinate. Spatial data with coordinate includes data which have position and geographical features such as river, roads and land form. Since the spatial data in GIS inspire of 
International Journal of Wireless \& Mobile Networks (IJWMN) Vol. 9, No. 1, February 2017

traditional methods store as digital, therefore updating, analysis, monitoring and editing of them will feasible with more speed and precision.

\subsection{GIS Components}

GIS apply four fundamental components about terrain data which are data input, data management, data analysis and output data.

\subsubsection{Data Input}

Data input component, convert data to usable form in GIS. Generally georeference data are in paper maps, table of descriptive information, electronic files of maps and descriptive information of them, aerial photos or satellite images. Data insertion may be easier than changing the file format or may be more complicated. Large databases creation may be have cost five or ten times of GIS hardware and software.

\subsubsection{Data Management}

Data management includes functions for storage, keeping and retrieval information inside of databases. There are different methods for data organization as files which computer could read them. Data structure is a method which data are organized based on it and files relation to each other will determine limitation on the information retrieval and speed of retrieval operation.

\subsubsection{Data Analysis}

The main goal in GIS is preparing the situation for spatial analysis. Therefore, information protection capability and map production is limited. In normal condition, spatial analysis could make easier the decisions making. Analysis is powerful component that GIS give to programmer and designer. Quantitative feature of GIS, give this capability to researches to use mathematics and statistics as a complementary. Analysis functions and works with data in GIS, is determinant of information that this system could creates.

\subsubsection{Output Data}

Data output in different GIS based on quality, precision and ease of use has a much more diversity than these system capabilities. Output data might be in different forms such as map or table of values or writing, and presents on the paper or digital. Required output functions will determine based on user requirement, therefore user interference is very important in determination of required outputs.

\subsection{Data Types in GIS}

One of the main difference of GIS with other informative systems is the nature of data used in this system that are recognize as georeference spatial data or coordinate spatial data that are belong to specified place and position on the ground. In GIS, information about situation or spatial phenomena is called spatial data and qualitative and quantitative characteristics of phenomena are called descriptive data. 


\subsection{Layers Combination}

In informative systems, data store in different tables. Available spatial data in spatial information systems for increasing their performance and operational capabilities will store in different layers. Each of the informative layers, store one of the data and these layers in interact with one another, provides capability of various analyses. Mostly for modeling and analysis, it is needed to combine different information layers. For combination of layers, there is different methods that most important of them is Overlap Index method.

\subsubsection{Overlap Index Method}

In overlap index model, in addition to applying weight of each single factor than other factors, each classes and spatial units in factors will weight based on relative importance and expert comments. After weighting operation, factors combine together according to equation (7).

$\mathrm{S}=\frac{\sum W_{i} S_{i j}}{\sum W_{i}}$

where $W_{i}$ is $i$-th map weight, $S_{i j}$ is $j$-th class of $i$-th map and $S$ is the value of each spatial unit in output map. This model have a more priority and flexibility than Boolean logic model, but linear nature and inability in determination of correct weighting variations relevant to border classes of each factor is disadvantages of it.

\subsection{Clustering}

Clustering defines as the data set classification process based on similarity to subsets named cluster. This classification will carry out such that member of each set had a more similarity together and more difference to other members. There are different techniques for data clustering. Among these methods, Agglomerative Hierarchical Clustering (AHC) is an appropriate method which without any pervious information of data pattern, categorization is possible. Hierarchical clustering algorithms display data as a tree, which is called Dendogram hierarchical tree.

Dendogram composed of nodes layers which each of them shows a cluster. To obtain similarities between data in hierarchical clustering, there is different methods that are Ward's method, Complete linkage, Simple linkage, Strong linkage, Flexible linkage, Unweighted pair-group average and Weighted pair-group average.

In this paper, Ward's method is used. This method uses a criterion to decrease outlier data losses. Ward's method uses the sum of squared difference between each data of cluster and mean vector of that cluster as a criterion for assessing a cluster. Ward's algorithm is as follows.

1. First each data will consider as a cluster.

2. For total possible clusters pair of clusters set, two clusters will select which sum of squared difference between cluster data of community of them and the mean cluster is minimum.

3. Two selected cluster will combine

4. Until number of clusters reach to intended number, steps 1,2 and 3 will repeat. 
Ward's method states that the distance between two clusters, A and B, how the distance of squares will increase when they combine [23]. The equation (8) shows the relation.

$$
\Delta(A, B)=\sum_{i \in A \cup B}\left\|\vec{x}_{i}-\vec{m}_{A \cup B}\right\|^{2}-\sum_{i \in A}\left\|\vec{x}_{i}-\vec{m}_{A}\right\|^{2}-\sum_{i \in B}\left\|\vec{x}_{i}-\vec{m}_{B}\right\|^{2}=\frac{n_{A} n_{B}}{n_{A}+n_{B}}\left\|\vec{m}_{A}-\vec{m}_{B}\right\|^{2}
$$

where $\vec{m}_{j}$ is the centre of cluster $j, n_{j}$ is the number of points in cluster $j$ and $\Delta$ is merging cost of combining the clusters $\mathrm{A}$ and $\mathrm{B}$.

\section{GeOSTATISTICS}

With use of geostatistic analysis, easily could create a map or continuous area of sampled points. Sampled values can be hauteur, temperature, air pollution and etc. Also geostatistic analysis could be used for spatial modeling in different applications. One of the advantages of this analysis is fitness of model area to sampled points. Geostatistic analysis is going to find the way for spatial continuously description and also statistic and deterministic tools collections and spatial variations modeling.

\subsection{Geostatistic Definition}

Geostatistic in European concept is a branch of statistics which data coordinate of under study population and consequently spatial structure of data will study. In classical statistic, generally samples which taken from population assume random. In other words, it assumes samples are independent of each other, therefore existence of one sample, will not provide any information about next sample. But in geostatistic, samples are not independent and according to this theory, adjacent samples to the certain distance have a spatial dependency. Therefore spatial position of samples with intended quantity value can be analyzed together. This spatial communication in mathematical formats will name spatial structure. Thus in geostatistical, variables will be discussed that they have spatial structure.

\subsection{Interpolation}

Sensors in WSNs have been placed in specific locations and they have ability to measure intended parameters in that point up to limited radius. Therefore these points information should convert to area information. One of the effective methods in this field is usage of interpolation techniques. According to the researches, geostatistics methods are one of the best methods for spatial data interpolation. There are different geostatistics methods which most important of them are: Inverse Distance Weight (IDW), Spline and Kriging. Between these methods Kriging is one of the best and most applicable for interpolation. Therefore in this paper for map creation, this interpolation method is used.

\subsubsection{Kriging Interpolation}

Generally, interpolation which carry out based on spatial statistical estimation models is process that it could be possible to obtain quantity in undefined coordinate with use of same quantity in other points with define coordinate. Most important spatial statistical estimator is Kriging. Kriging is an estimator method which is based on weighted moving average and it is the best unbiased estimator. Equation (9) shows the kriging equation. 
$\hat{Z}=\sum_{i=1}^{n} \lambda_{i} Z_{i}$

where $\hat{Z}$ is an approximated parameter, $\lambda_{i}$ is a weight or importance quantity related to $i$-th sample and $Z_{i}$ is a specified parameter. This type of kriging is linear, because it is linear combination of $n$ data. For using this estimator, $Z$ variable should have a normal distribution otherwise nonlinear kriging should be used or normalized the variable with usage of statistical conversions. Kriging estimator has two brilliant features. Firstly it doesn't have systematic error. Secondly it has minimum estimation variance.

Necessities of first condition establishment is average of estimation error be zero. Therefore it can be written as equation (10).

$$
E\left(Z_{i}-\sum_{i=1}^{n} \lambda_{i} Z_{i}\right)=0
$$

By simplification of equation (10), equation (11) is as below.

$$
E\left(Z_{i}\right)-E\left[\sum_{i=1}^{n} \lambda_{i} Z_{i}\right]=0
$$

Also $E\left(Z_{i}\right)=m$, therefore $m-\sum_{i=1}^{n} \lambda_{i} m=0$

Because $m \neq 0$, thus equation (12) must establish.

$$
\sum_{i=1}^{n} \lambda_{i}=1
$$

Therefore to minimize kriging estimate variance, it is necessary to minimize it in term of kriging indices. There are different methods for kriging which are simple kriging, ordinary kriging, token kriging, probabilistic kriging and clustered kriging [24, 25].

\section{IMPLEMENTATION}

The study area in this paper is Grand Saint Bernard Pass with 2400 meter length in border of Switzerland and Italy. This passage is in the mountain ranges between two countries which from north lead to Switzerland and from south lead to Italy. The studied WSN in this paper is the established WSN around the Grand Saint Bernard Pass named SensorScope which is implemented with EPFL University in 2007. Data of this sensor network download from web site with address: http://lcav.epfl.ch/page-86035-en.html. This WSN has 23 sensors which measure environmental data such as ambient temperature, land surface temperature, sun radiation, relative humidity, soil humidity, speed and direction of wind.

\subsection{Data Collection and Preparation}

To evaluate and locating of sensors in WSN, effective criteria should identified and their impact to be studied. Table 3 shows the most important effective parameters and their impact. According to effective criteria, required data must be collected and prepare for usage in ArcGIS software. For calculation and analysis, types of all information layers in ArcGIS software will raster. In raster model, whole map area divide to network of tiny and regular cells that named pixel. In fact, 
raster type is $n \times m$ arrays of pixels. Each of these pixels is indicator of one position in the earth area and corresponds to spatial range in real world. In each pixel, a numerical value will save which indicates nature of that place in real world that is called Digital Number (DN).

Table 3. Most important parameter with their impact on WSN performance

\begin{tabular}{|l|l|}
\hline Criteria Name & Impact of criteria on WSN performance \\
\hline DEM & Whatever hauteur is higher, the network performance is lower \\
\hline Sensor coverage & Whatever coverage is less, measuring cavity is more \\
\hline Sensor & Whatever communication range is less, network performance decreases \\
\hline Sensor energy & Sensors energy has a more effect on the network performance \\
\hline Scalability & More scalability increase the network performance \\
\hline
\end{tabular}

The reason for usage of raster format instead of vector format is that calculation and overlap analysis in raster model is easier. Station coordinate and features of them is prepared in Excel format. Then this file is inserted into GIS environment and layer is created. The contents of this layer are shown in table 4. This table includes station coordinate in UTM and WGS-84, hauteur, coverage, energy and communication range of stations

\subsection{Classification}

\subsubsection{DEM}

Required DEM for study area is obtained from Earth Data website which belongs to NASA and included 30 meter DEM of whole earth [26]. This region is in Europe with N45E008. Figure 2 shows a categorized DEM of region which is used in this paper. As it shown in figure 2, different hauteur has different colors which are classified in five classes. Low hauteur has a white color and high hauteur is darker. These DEM data are prepared in a raster format which will use in GIS and ArcGIS software for continuation of simulation in the paper.

Table 4. Data collection from 23 stations in studied area

\begin{tabular}{|l|l|l|l|l|l|l|l|l|}
\hline Obiect- & Latitude & longitude & $\mathbf{X}$ & $\mathbf{Y}$ & Hauteur & Coverage & Energv & Communication \\
\hline 1 & 45.8688 & 7.17348 & 358235.1 & 5081092.6 & 2430 & 50 & 1 & 100 \\
\hline 2 & 45.86887 & 7.17252 & 358160.8 & 5081102.1 & 2446 & 50 & 1 & 100 \\
\hline 3 & 45.86924 & 7.17162 & 358091.9 & 5081144.8 & 2466 & 50 & 3 & 100 \\
\hline 4 & 45.87159 & 7.18016 & 358760.6 & 5081390.7 & 2316 & 50 & 3 & 100 \\
\hline 5 & 45.87014 & 7.17806 & 358594 & 5081233.3 & 2358 & 50 & 2 & 100 \\
\hline 6 & 45.86923 & 7.17721 & 358525.7 & 5081133.7 & 2394 & 50 & 2 & 100 \\
\hline 7 & 45.86809 & 7.17522 & 358368.4 & 5081010.6 & 2424 & 50 & 3 & 100 \\
\hline 8 & 45.87177 & 7.1814 & 358857.3 & 5081408.5 & 2324 & 50 & 3 & 100 \\
\hline 9 & 45.8702 & 7.17928 & 358688.8 & 5081237.8 & 2368 & 50 & 3 & 100 \\
\hline 10 & 45.8707 & 7.17835 & 358617.9 & 5081295 & 2370 & 50 & 3 & 100 \\
\hline 11 & 45.86974 & 7.17517 & 358368.7 & 5081194 & 2408 & 50 & 2 & 100 \\
\hline 12 & 45.87081 & 7.17937 & 358697.4 & 5081305.4 & 2339 & 50 & 1 & 100 \\
\hline 13 & 45.86933 & 7.1755 & 358393.2 & 5081147.9 & 2383 & 50 & 2 & 100 \\
\hline 14 & 45.86939 & 7.17682 & 358495.8 & 5081152.2 & 2378 & 50 & 1 & 100 \\
\hline 15 & 45.87262 & 7.18097 & 358826.1 & 5081503.7 & 2290 & 50 & 2 & 100 \\
\hline 16 & 45.87249 & 7.18013 & 358760.6 & 5081490.7 & 2317 & 50 & 3 & 100 \\
\hline 17 & 45.87355 & 7.18121 & 358847.1 & 5081606.6 & 2280 & 50 & 3 & 100 \\
\hline 18 & 45.86923 & 7.17489 & 358345.6 & 5081137.8 & 2391 & 50 & 3 & 100 \\
\hline 19 & 45.87058 & 7.15495 & 356801.5 & 5081323.4 & 2416 & 50 & 3 & 100 \\
\hline 20 & 45.86954 & 7.15339 & 356677.8 & 5081210.7 & 2375 & 50 & 3 & 100 \\
\hline 21 & 45.86992 & 7.15264 & 356620.5 & 5081254.2 & 2407 & 50 & 3 & 100 \\
\hline 22 & 45.87004 & 7.1544 & 356757.4 & 5081264.4 & 2394 & 50 & 3 & 100 \\
\hline 23 & 45.86984 & 7.15608 & 356887.3 & 5081239.2 & 2405 & 50 & 3 & 100 \\
\hline
\end{tabular}




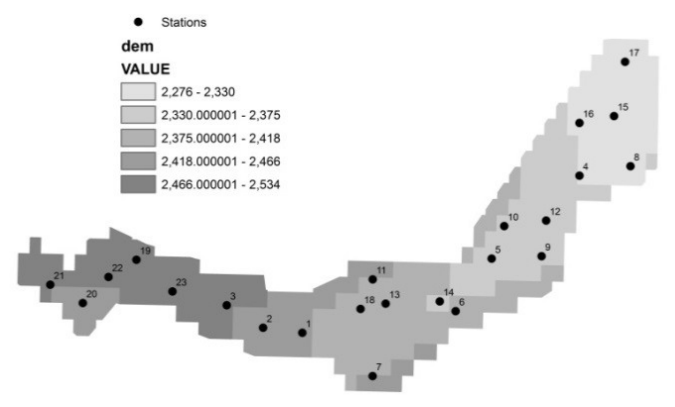

Figure 2. DEM of studied area

\subsubsection{Coverage range}

The coverage range of sensor in simulation is 50 meter and sensor measurability is considered 50 meter. To model the coverage range, the buffering feature of GIS is used. Buffering in GIS is in the spatial analysis. Figure 3 shows a buffering map of sensors (coverage of sensors). In figure 3, white color means better coverage and darker color becomes fewer converges.

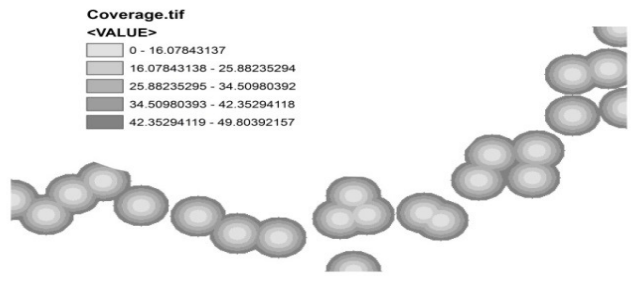

Figure 3. Coverage map of network sensors

\subsubsection{Communication Range}

Sensor communication range is considered 100 meters. For communication range, same as coverage, buffering is used and then the map of it is shown in figure 4. Same as figure 3, in figure 4 , white colour means better communication and darker color becomes less communication.

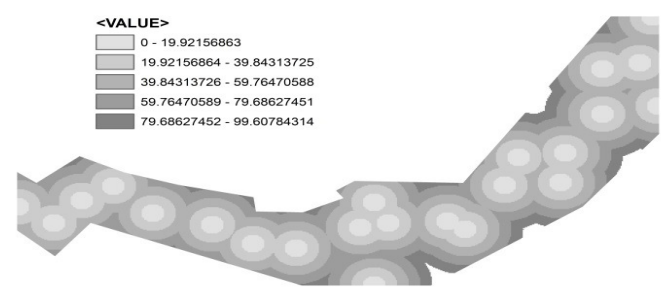

Figure 4. Sensor communication range map

\subsubsection{Energy Consumption}

Energy consumption has an importance in WSN. Sensors of SensorScope network have 3-state for energy consumption, State 1 - using a constant battery, State 2 - rechargeable batteries and State 3 - solar panel. 
The idealist one is using these three states. In the studied network at this paper, some sensors have batteries as state one, some have power provision as state two and some include all three states for power generation. However the sensor uses a more sustainable energy, measurement around it will carry out with more stability. Therefore to provide map of energy consumption of area, sensors are classified to three categories and according to it, map of network energy consumption will produce. Number one in this classification means lower energy stability and number three means highest energy stability. To calculate network energy consumption map, interpolation methods is used. One of the effective methods is Kriging interpolation that is used in this paper for preparing energy consumption map. Interpolation methods in GIS are as statistical analysis of geostatic. Energy consumption map of studied area with use of Kriging interpolation is shown in figure 5. Darker color has a lower energy and white color has a higher energy.

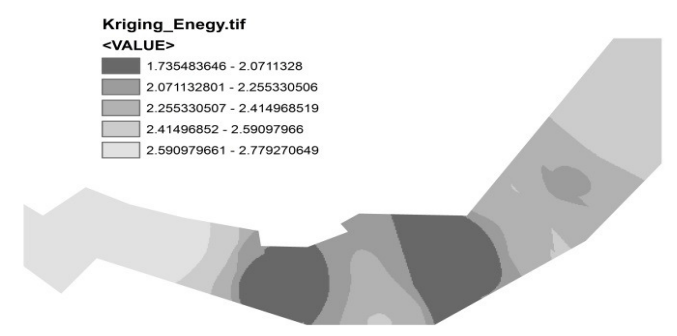

Figure 5. Energy consumption map of studied area

\subsubsection{Scalability}

Network scalability is a withstanding of network against variations. To prepare scalability map in different points of network, first scalability of each sensor should be calculated and then with use of interpolation methods, it will extend to the others and obtain a scalability map. To count number of sensors in each sensor range for scalability, spatial joint is used which will create a field includes number of sensors as normalized in Table 5. Finally to obtain scalability for whole points in the area like as energy consumption map, kriging interpolation is used. Scalability map is calculated for studied area and shown in figure 6. Scalability in stations seven and seventeen is zero which is a darkest region in the map.

Table 5. Normalized network scalability in each sensor

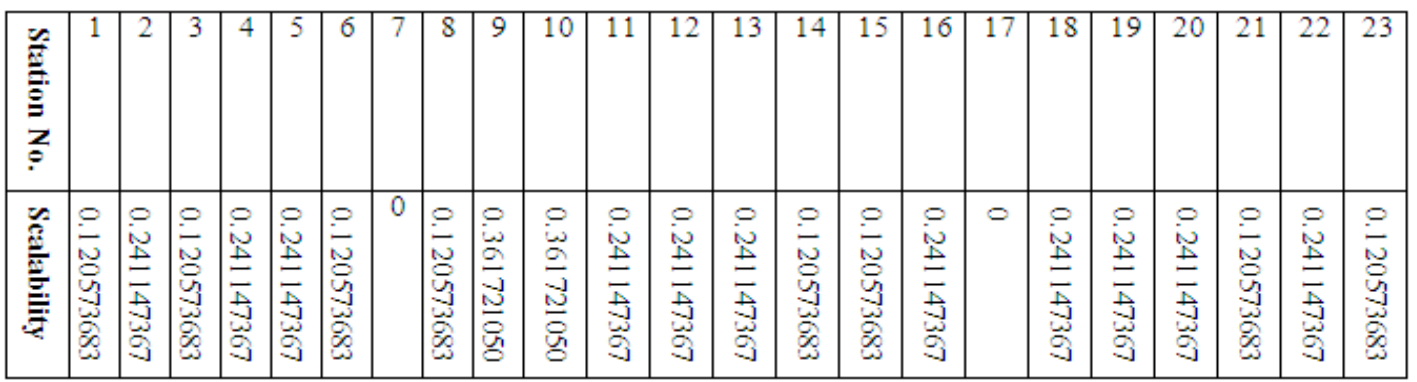




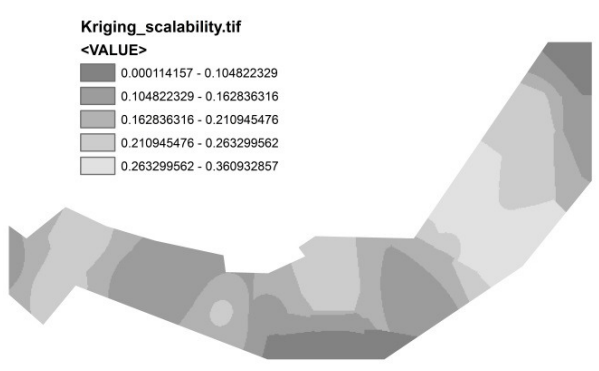

Figure 6. Scalability map of studied area

\subsection{Reclassification}

The maps that are prepared in GIS are called layer. The prepared layers are in the raster format. A raster layer with respect to its resolution is made with some isometric pixels which each of them has its own numerical value. The operation of homogenization and clustering of data in rasterized layer will call reclassification. With use of reclassification, after separating pixels worth in classes, a rasterized layer will produce. With combination of rasters in one class, homogeneous classes will make. In reclassification, number of classes, distance between classes and numerical worth of each class will determine. Each produced maps will classify in five class, and value of each class with respect to effect of it on the WSN is valued one to five that number one has lowest worth and number five has highest worth. At continuation, reclassification for each map is shown.

\subsubsection{DEM}

However hauteur is more, the communication between sensors is harder. Therefore in reclassification of DEM, classes with higher hauteur will have lower worth. Table 6 shows a reclassification of DEM in GIS.

Table 6. Reclassification of DEM in GIS

\begin{tabular}{|l|l|l|l|l|l|}
\hline Old values & $2276-2330$ & $2330-2375$ & $2375-2418$ & $2418-2466$ & $2466-2534$ \\
\hline New values & 5 & 4 & 3 & 2 & 1 \\
\hline
\end{tabular}

In figure 7 output of DEM reclassification is shown. Higher hauteur has a darker color, which means is not worthy situation for WSN.

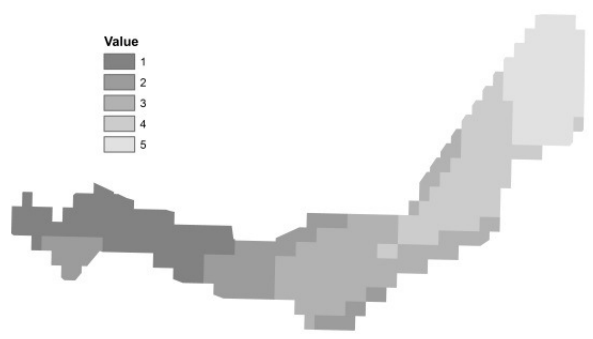

Figure 7. Reclassification output of DEM 


\subsubsection{Coverage Range}

In coverage reclassification, the aim is whole region in the studied area lay inside the coverage range. Hence the regions inside of coverage range have maximum worth five and regions outside of coverage range have a minimum value zero. Therefore sensor coverage range map will classify into two classes. Figure 8 shows a reclassification output of sensor coverage range map that white colour is points within and black color is point out of coverage range.

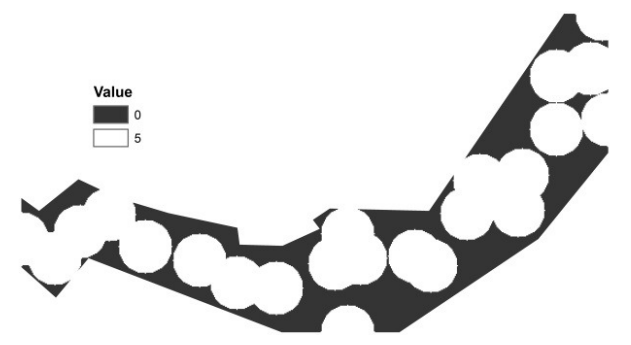

Figure 8. Reclassification output of sensor coverage range map

\subsubsection{Communication Range}

Reclassification of communication range map is similar to coverage range map and it has to classes. Class 0-100 meter has most worth five and outside of this range has a least worth zero. Figure 9 shows a reclassification for communication range. As shown in figure 9, most study area is inside of sensor communication range and only regions with dark colors are cavity communication.

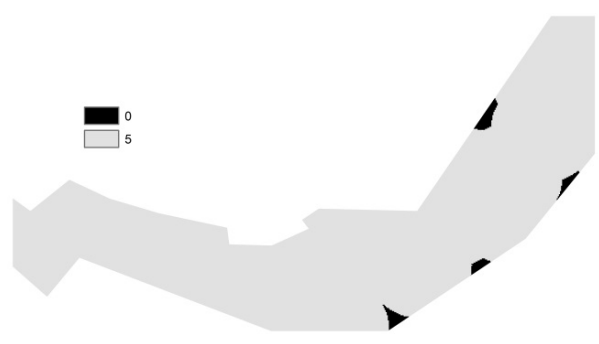

Figure 9. Output map of reclassification communication range

\subsubsection{Power Consumption}

As explained in section 6.2.4, however number assigned to the energy consumption in energy map is higher; it means that region has more stability, therefore in reclassification energy consumption map; higher classes have a more worth. In figure 10, output map of reclassification for energy consumption is shown. Whatever the colours are brighter, energy consumption in the region is better. 


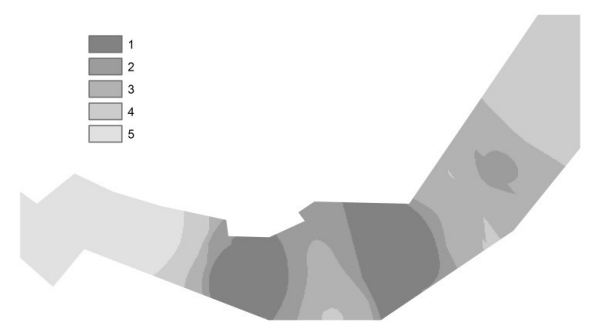

Figure 10. Output map of reclassification energy consumption

\subsubsection{Scalability reclassification}

However scalability in each region of network is higher, the network will be more stable. Hence for scalability reclassification map, higher classes have more worth. Figure 11 shows output map for scalability reclassification. As shown in figure 11, brighter color means more scalability.

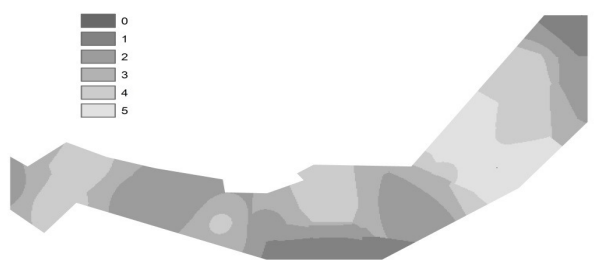

Figure 11. Output map of scalability reclassification

\subsection{Calculation of Criteria Weights}

After selecting criteria and collecting data, implementation and sensor placement process will carry out. To improve a performance of WSN, the criteria should be weighted. These weights will acquire with experts in the WSN field. Then with use of AHP method, in the expert choice software, the optimum placement for sensor will obtain. Finally with use of Ordered Weighted Averaging (OWA) method, layers in ArcGIS software will combine and places that they require to place new sensor on them will determine. After providing, preparing and reclassification of criteria map, these maps should be combined together to determine regions which require to install new sensors. For considering the weight for each criterion, multifactor decision methods should be used. One of the best and effective methods is AHP. To implement AHP method, some questionnaire prepared and experts in WSN field, with comparison of each two criteria together, they determined importance value of each criterion. Then the answers to these questions were inserted to the expert choice software to get a weight of each criterion. Table 7, shows pair wise comparison and inconsistent coefficient which has obtained from questionnaire.

Table 7. Pair wise comparison and inconsistent coefficient

\begin{tabular}{|l|l|l|l|l|l|}
\hline Criterion & Energy & DEM & Scalability & Coverage & Range \\
\hline Energy & & 3.0 & 3.0 & 5.0 & 5.0 \\
\hline DEM & & & 5.0 & 7.0 & 3.0 \\
\hline Scalability & & & & 3.0 & 5.0 \\
\hline Coverage & & & & & 5.0 \\
\hline Range & Incon : 0.10 & & & & \\
\hline
\end{tabular}


As it shown in Table 7, the inconsistent coefficient is 0.10 which is in the allowable limit for this coefficient.

\subsection{Combination of criteria map and preparing final map}

Table 8 shows a final weight for each criterion. After determination of criteria importance, with use of hierarchical analysis process, the criteria should combine together to determine the locations that require new sensor establishment. Output map is obtained from criteria map combination such that each pixel shows a value to determine the suitability of the location where the new sensor is placed with respect to all effective factors. Considering that the criteria used in different ranges have different worth; therefore in this paper with use of overlap index method for criteria map combination, the output is calculated and shown in figure 12. The darker area in figure 12 means installation of new sensor in that region is more necessary. Therefore five sensors were installed in the regions which they sever need to the sensor installation. Figure 13 shows locations of new sensors (sensors 24 to 29).

Table 8. Final weight calculated for each criterion with AHP method

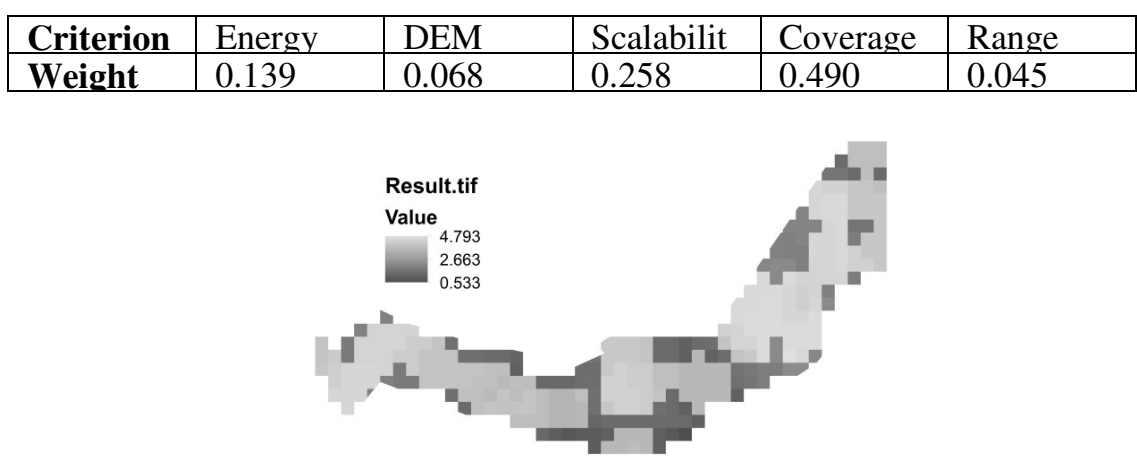

Figure 12. Criteria combination output with overlap index method

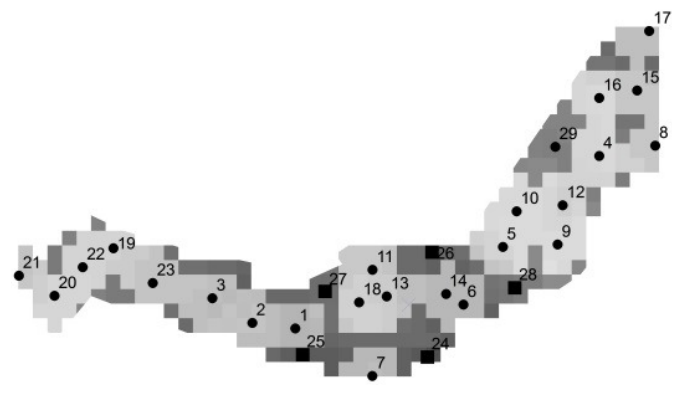

Figure 13. New sensor installation map

After installation of these new sensors, all implementation steps have been carried out and results are shown in figure 14. As it is clear in this figure, after adding some new sensors in the dark region which had low performance, network performance is increased. 


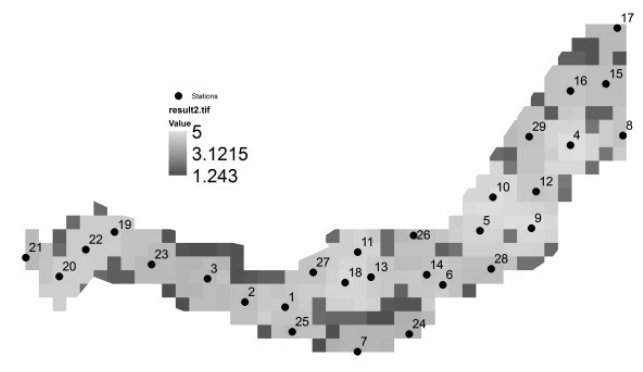

Figure 14. New sensor locations based on output of overlap index method

Table 9 shows a pixel value in each station before and after adding sensors and also the amount of network average performance improvement. As it is clear, network performance is increased averagely $21.11 \%$. This performance improvement for the new sensors installation is considerable.

Table 9. Performance improvement for sensor placements

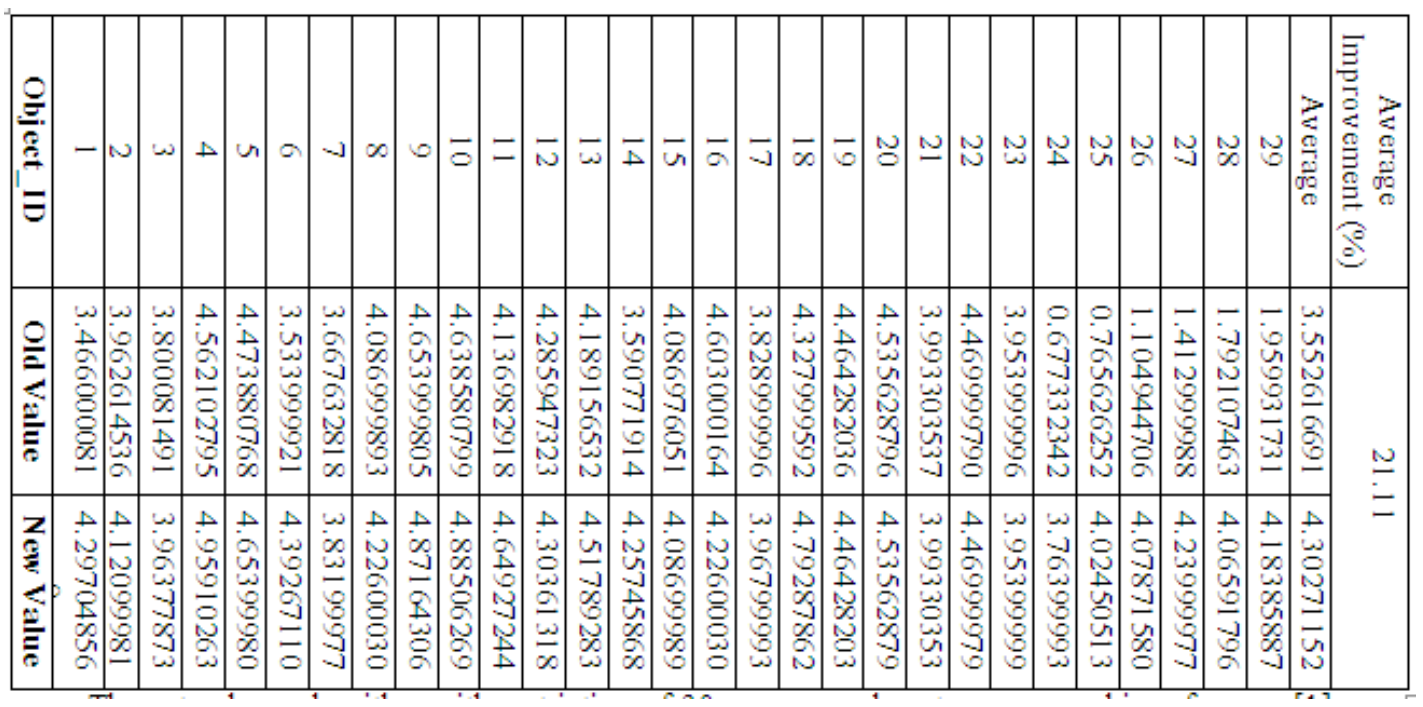

The ant colony algorithm with restriction of 30 sensors and cost as proposed in reference [1] implemented in this study area. Also the proposed method of reference [4] which considers only coverage parameter (same as our propose method in section $6.2 .2(50 \mathrm{~m})$ ) is implemented. Also ANOVA method is implemented as stated in [6] with DEM parameter as section 6.2.2. Bee algorithm is also use for sensor placement to get the best coverage (section 6.2.2) as reference [7]. In reference [8] which spatial information is used is also implanted for the studied area section 6. Table 10 shows the performance improvement for these methods. As it is clear the proposed method has a better performance improvement in comparison to other proposed methods. The main reason which proposed method in this paper has a better improvement is that it considers all of the important parameters together with proper weight for sensor placement which other methods only consider some of these parameters for network performance improvement. Also in Table 10, the percentage of added number of sensors is written which proposed method has a lowest percentage of added sensors with $26.09 \%$. 
International Journal of Wireless \& Mobile Networks (IJWMN) Vol. 9, No. 1, February 2017

Table 10. Performance Comparison of Different Methods

\begin{tabular}{|c|c|c|c|c|c|c|c|}
\hline$\underset{\Xi}{\stackrel{\Xi}{\Xi}}$ & 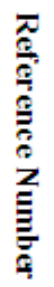 & 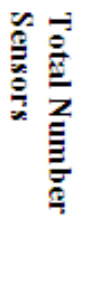 & 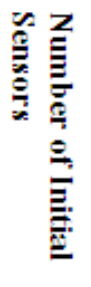 & $\frac{2}{\frac{a}{6}}$ & 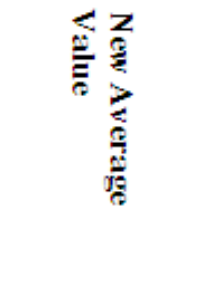 & 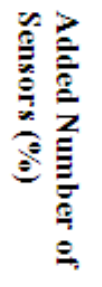 & 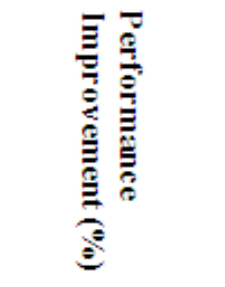 \\
\hline Colony & 1 & 30 & \multirow{6}{*}{23} & \multirow{6}{*}{ 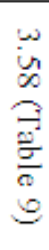 } & 4.11 & 30.43 & 14.89 \\
\hline Coverage & 4 & 35 & & & 3.95 & 52.17 & 10.45 \\
\hline ANOVA & 6 & 32 & & & 4.15 & 39.13 & 15.87 \\
\hline Bee & 7 & 34 & & & 4.10 & 47.83 & 14.67 \\
\hline Spatial & 8 & 31 & & & 4.23 & 34.78 & 18.32 \\
\hline Proposed & -- & 29 & & & 4.28 (Table 9) & 26.09 & 21.11 (Table 9) \\
\hline
\end{tabular}

For better clearance in figures 15 and 16 the bar chart of the results are shown.

Performance improvement percentage of compar edmethods $(\%)$

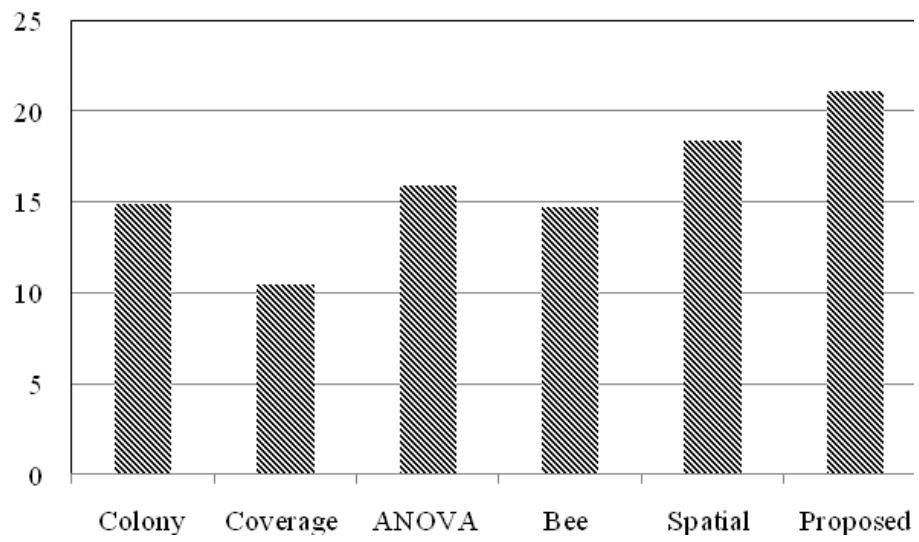

Figure 15. Performance improvement percentage of different methods

Perecentage of added number of sensors (\%)

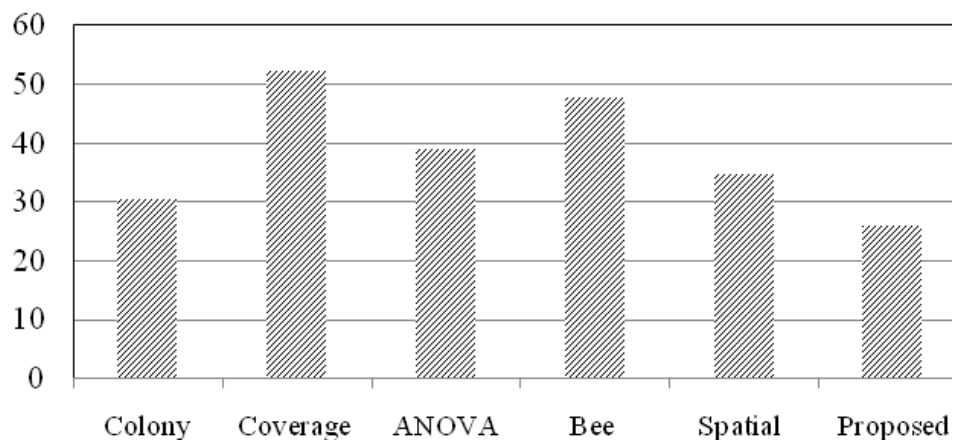

Figure 16. Percentage of added number of sensors 
International Journal of Wireless \& Mobile Networks (IJWMN) Vol. 9, No. 1, February 2017

\section{Conclusions}

In this paper, first most important and effective parameters which have effect on the network are studied that these parameters are: coverage, communication range, scalability and DEM. After determination of effective criteria, required data are prepared to insert to GIS. In fact GIS creates an environment to combine and carry out different analysis on data. After insertion data into GIS, with use of AHP method, to compare different criteria with themselves, relative worth of each criterion is determined. At the end, with use of analysis and toolboxes in GIS, these criteria are combined together and places which require a new sensor installation are determined. Results shown whenever hauteur is more, connections between sensors are harder and related region requires more sensor installation. The performance of proposed method, will improve $21.11 \%$ with sensor placement in the low performance locations. Also the percentage for number of added sensor is $26.09 \%$ that is lowest number between others compared methods.

\section{REFERENCES}

[1] Miri S. M. \& Afshar A., (2014) "Optimum Layout for Sensors in Water Distribution Networks through Ant Colony Algorithm: A Dual use Vision", Water and Sewage Quarterly, Vol. 25, No. 3, pp $67-75$.

[2] Ashrafee Kh., Ghader S., Esfehanian V. \& Motasadi S., (2007) "Air Pollution Measurement Layout in Large Tehran", Journal of Environmental Study, Vol. 33, No. 44, pp 1-10.

[3] Bahrampour M. \& Bemaniam M. R., (2012) "Study on Optimum Location of Disaster Management Sites with use of GIS", Journal of Emergency Management, Vol. 1, No. 1, pp 51-59.

[4] Osais, Y.E., M. St-Hilaire \& R.Y. Fei, (2010), "Directional sensor placement with optimal sensing range, field of view and orientation", Mobile Networks and Applications, Vol. 15, No. 2, pp 216-225.

[5] Isovitsch, S.L. \& J.M. VanBriesen, (2008), "Sensor placement and optimization criteria dependencies in a water distribution system", Journal of Water Resources Planning and Management, Vol. 134, No. 2, pp 186-196.

[6] Castello, C.C. \& Fan J., (2010), "Optimal sensor placement strategy for environmental monitoring using wireless sensor networks", 42nd Southeastern Symposium on System Theory (SSST), pp 275279.

[7] Mini, S., S.K. Udgata \& S.L. Sabat, (2014), "Sensor deployment and scheduling for target coverage problem in wireless sensor networks", IEEE sensors journal, Vol. 14, No. 3, pp 636-644.

[8] Argany, M., (2015), Development of a GIS-Based Method for Sensor Network Deployment and Coverage Optimization, $\mathrm{PhD}$ Thesis, Université Laval.

[9] M. Marks, (2010), "A survey of multi-objective deployment in wireless sensor networks", Journal of Telecommunications and Information Technology, pp 36-41.

[10] C.Zhu, C. Zheng, L. Shu \& G. Han, (2012),"A survey on coverage and connectivity issues in wireless sensor networks", Journal of Network and Computer Applications, Vol. 35, No. 2, pp 619-632.

[11] S.Megerian, F. Koushanfar, M. Potkonjak \& M.B. Srivastava, (2005), "Worst and best-case coverage in sensor networks", IEEE transactions on mobile computing, Vol. 4, No. 1, pp 84-92.

[12] S.R.Angajala, (2012), "Coverage Problems in Sensor Networks", IJECCE, Vol. 3, No. 1, pp 104-110.

[13] A. Ghosh \& S.K. Das, (2006), "Coverage and connectivity issues in wireless sensor networks", Mobile, wireless, and sensor networks: Technology, applications, and future directions, pp 221-256.

[14] K.Chakraborty, S.S. Iyengar, H. Qi \& E. Cho, (2002), "Grid coverage for surveillance and target location in distributed sensor networks", IEEE transactions on computers, Vol. 51, Vol. 12, pp 14481453.

[15] D.S. Deif \& Y. Gadallah, (2014), "Classification of wireless sensor networks deployment techniques", IEEE Communications Surveys \& Tutorials, Vol.16, No. 2, pp 834-855.

[16] M. Leoncini, G. Resta \& P. Santi,(2009), "Partially controlled deployment strategies for wireless sensors", Ad Hoc Networks, Vol. 7, No. 1, pp 1-23.

[17] F.Bouabdallah, N. Bouabdallah \& R. Boutaba, (2009), "On balancing energy consumption in wireless sensor networks", IEEE Transactions on Vehicular Technology, Vol. 58, No. 6, pp 2909-2924. 
International Journal of Wireless \& Mobile Networks (IJWMN) Vol. 9, No. 1, February 2017

[18] F.Ishmanov, A.S. Malik \& S.W. Kim, (2011), "Energy consumption balancing (ECB) issues and mechanisms in wireless sensor networks (WSNs): a comprehensive overview", European Transactions on Telecommunications, Vol. 22, No. 4, pp 151-167.

[19] L.K.Alazzawi, A. M. Elkateeb \& A. Ramesh, (2008), "Scalability analysis for wireless sensor networks routing protocols". International Conference on Advanced Information Networking and Applications-Workshops (AINAW), pp 139-144.

[20] M.Vuckovik, D. Trajanov \& S. Filiposka, (2010), "Durkin's propagation model based on triangular irregular network terrain", In International Conference on ICT Innovations, Springer.

[21] R.Shorey, A. Ananda, M.C. Chan \& W.T. Ooi, (2006), Mobile, wireless, and sensor networks: technology, applications, and future directions. John Wiley \& Sons.

[22] E. Triantaphyllou \& S. H. Mann, (1995), "Using the analytic hierarchy process for decision making in engineering applications: some challenges", Vol. 2, No. 1, pp 35-44.

[23] P. Berkhin, (2006), A survey of clustering data mining techniques, in Grouping multidimensional data, Springer. pp 25-71.

[24] N. Cressie, (1990), "The origins of kriging", Mathematical geology, Vol. 22, No. 3, pp. 239-252.

[25] Oliver, M.A. \& R. Webster, (1990), "Kriging: a method of interpolation for geographical information systems", International Journal of Geographical Information System, Vol. 4, No. 3, pp 313-332.

[26] EarthData availabe from: dwtkns.com/srtm30m.

\section{Author}

Gholamreza Farahani received his BSc degree in electrical engineering from Sharif University of Technology, Tehran, Iran, in 1998 and MSc and PhD degrees in electrical engineering from Amirkabir University of Technology (Polytechnic), Tehran, Iran in 2000 and 2006 respectively. Currently, he is an assistant professor in the Institute of Electrical and Information Technology, Iranian Research Organization for Science and Technology (IROST), Iran. His research interest is Wireless Network especially Wireless Sensor network.

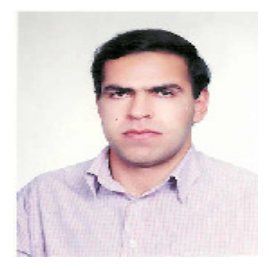

